\title{
Genetic Variations and mRNA Expression of NRF2 in Parkinson's Disease
}

\author{
Caroline Ran, ${ }^{1}$ Karin Wirdefeldt, ${ }^{2,3}$ Lovisa Brodin, ${ }^{2}$ Mehrafarin Ramezani, ${ }^{1}$ \\ Marie Westerlund, ${ }^{4}$ Fengqing Xiang, ${ }^{5}$ Anna Anvret, ${ }^{1}$ Thomas Willows, ${ }^{2}$ Olof Sydow, ${ }^{2}$ \\ Anders Johansson, ${ }^{2}$ Dagmar Galter, ${ }^{1}$ Per Svenningsson, ${ }^{2}$ and Andrea Carmine Belin ${ }^{1}$ \\ ${ }^{1}$ Department of Neuroscience, Karolinska Institutet, 17177 Stockholm, Sweden \\ ${ }^{2}$ Department of Clinical Neuroscience, Karolinska Institutet, 17177 Stockholm, Sweden \\ ${ }^{3}$ Department of Medical Epidemiology and Biostatistics, Karolinska Institutet, 17177 Stockholm, Sweden \\ ${ }^{4}$ Department of Neurobiology, Care Sciences and Society, Karolinska Institutet, 14183 Huddinge, Sweden \\ ${ }^{5}$ Department of Women's and Children's Health, Karolinska Institutet, 17176 Stockholm, Sweden
}

Correspondence should be addressed to Caroline Ran; caroline.ran@ki.se

Received 18 November 2016; Revised 6 April 2017; Accepted 12 April 2017; Published 2 May 2017

Academic Editor: Hélio Teive

Copyright (C) 2017 Caroline Ran et al. This is an open access article distributed under the Creative Commons Attribution License, which permits unrestricted use, distribution, and reproduction in any medium, provided the original work is properly cited.

Nuclear factor erythroid 2-like 2 (NRF2) encodes a transcription factor regulating mechanisms of cellular protection and is activated by oxidative stress. NRF2 has therefore been hypothesized to confer protection against Parkinson's disease and so far an NRF2 haplotype has been reported to decrease the risk of developing disease and delay disease onset. Also NRF2 adopts a nuclear localization in Parkinson's disease, which is indicative of increased NRF2 activity. We have investigated the association between NRF2 and Parkinson's disease in a Swedish case-control material and whether NRF2 expression levels correlate with NRF2 genetic variants, disease, or disease onset. Using pyrosequencing, we genotyped one intronic and three promoter variants in 504 patients and 509 control subjects from Stockholm. Further, we quantified NRF2 mRNA expression in EBV transfected human lymphocytes from patients and controls using quantitative real-time reverse transcription PCR. We found that one of the promoter variants, rs35652124, was associated with age of disease onset $\left(\mathrm{X}^{2}=14.19, p\right.$ value $\left.=0.0067\right)$. NRF2 mRNA expression levels however did not correlate with the rs35652124 genotype, Parkinson's disease, or age of onset in our material. More detailed studies on NRF2 are needed in order to elucidate how this gene affects pathophysiology of Parkinson's disease.

\section{Introduction}

Parkinson's disease (PD) is the second most common neurodegenerative disorder in the aging population; approximately 20,000 people are affected by PD in Sweden which has a population around 9.6 million. PD is characterized by degeneration of dopamine (DA) neurons in substantia nigra $(\mathrm{SN})$, but there is degeneration of other neurons as well. Although there is a relatively large body of knowledge on the pathology of $\mathrm{PD}$, there is little understanding of the etiology. The existing treatment for PD today, for example, replacing DA, improving the effect of DA, or inhibiting the breakdown of DA, does not cure the disease but only reduces the symptoms. In order to develop better ways of combating
PD or better still prevent clinical symptoms altogether, one must decipher the causes of disease.

Typically, candidate genes for PD do not specifically relate to the DA system. Instead, they tend to serve general cellular purposes, such as to protect against reactive molecules or participate in mitochondrial function [1]. One gene that has been suggested to confer protection against $\mathrm{PD}$ is the nuclear factor erythroid 2-like 2 (NRF2) (OMIM 600492), on chromosome 2q31. It belongs to a family of genes together with NFE2 (OMIM 601490) and NFE2L1 (OMIM 163260), encoding basic leucine zipper (bZIP) transcription factors [2]. In normal conditions, NRF2 is not active; it resides in the cytoplasm bound to KEAP1 (kelch-like ECH association protein 1), a ubiquitin E3 ligase complex, which mediates 
TABLE 1: Demographics of the study population.

\begin{tabular}{lccccc}
\hline & Participants, $n$ & $\begin{array}{c}\text { Females, } \\
n(\%)\end{array}$ & $\begin{array}{c}\text { Mean age at } \\
\text { enrolment, years }\end{array}$ & $\begin{array}{c}\text { Mean age at } \\
\text { diagnosis, years }\end{array}$ & $\begin{array}{c}\text { Heredity, } \\
n(\%)\end{array}$ \\
\hline Controls & 509 & $290(57.3)$ & $71^{\star}$ & NA & NA \\
PD & 501 & $184(36.7)$ & 67.2 & 59.5 & $88(28.5)^{\star \star}$ \\
\hline
\end{tabular}

PD: Parkinson's disease; NA: not applicable; ${ }^{\star}$ age was unknown for 95 blood donors; ${ }^{\star \star}$ estimation based on 319 individuals for which this information was known.

NRF2 degradation by the proteasome. In response to oxidative stress, NRF2 is released from KEAP1 and translocated from the cytoplasm into the nucleus where it transactivates expression of genes with antioxidant activity, detoxifying enzymes, cell survival, and anti-inflammatory factors [3]. Expression of $N r f 2$, the rat orthologue of NRF2, decreases with increasing age and is accompanied by a drop in activity of phase II antioxidant enzymes, which are upregulated by NRF2 [4-6]. Subsequently, the decrease in NRF2 expression results in increased sensitivity to oxidative damage.

In PD, nuclear localization of NRF2 has been reported to be strongly induced in nigral neurons, but this response may be insufficient to protect neurons from degeneration [7]. Five genetic association studies have been published on NRF2 in relation to PD with variable results [8-12]. In 2010, von Otter et al. performed a genetic study in a Swedish and a Polish PD material and identified a haplotype including three promoter single nucleotide polymorphisms (SNPs) and five tag SNPs that was associated with decreased risk of PD as well as later age of onset of PD [11]. The association with delayed disease onset was later replicated in an extended study comprising more than $1000 \mathrm{PD}$ patients [12]. Another replication study on a subset of these SNPs in a Taiwanese PD population could not confirm these findings [8]. The importance of NRF2 SNPs has also been assessed in a Chinese PD population in which an association was discovered with two exonic SNPs but not with the promoter SNPs suggested by von Otter et al. $[9,11]$. Yet another SNP was found to be associated with PD risk in an Australian sample and, in this study, several SNPs and haplotypes were also found to affect the age of onset [10]. What is more, haplotype association has also been reported between NRF2 and Alzheimer's disease (AD) as well as with age-related cataract and amyotrophic lateral sclerosis (ALS) $[13,14]$. Overall it seems likely that genetic variants in NRF2 affect both the risk of developing neurodegenerative disorders such as PD and the age of onset, but as different sets of SNPs were included in these studies, as well as the differential distribution of haplotypes in populations with different ethnicity, it is difficult to pinpoint what combination of SNPs is responsible for the reported associations.

NRF2 may play an important role in cellular protection in neurodegenerative diseases as well as being a viable therapeutic target in the future. As described above, there are several indications supporting the involvement of NRF2 in neurodegenerative disorders. In the present study, we investigated four genetic variants in NRF2, rs35652124 (A>G), rs6706649 (G>A), rs6721961 (C>A), and rs2001350 (A>G), and their possible association with $\mathrm{PD}$ in our large homogenous Swedish case-control material in order to expand on the knowledge on NRF2 genetic variations as a risk factor in PD. The SNPs included in our study have previously been reported to be associated with PD in a Caucasian material consisting of Swedish and Polish individuals [11]. We additionally wanted to examine whether the NRF2 gene is differently expressed in patients and controls or affecting age of onset and if genetic variants identified to be associated with PD in this study affected expression in vivo.

\section{Materials and Methods}

2.1. Patients and Controls. PD patients were recruited to the study during routine outpatient visits to the Neurology clinic at Karolinska University Hospital, Stockholm, Sweden. Patients $(n=504)$ were unrelated of Swedish origin, mean age was 67.2 years, age at diagnosis was 59.4 years, and $36.7 \%$ were females (Table 1). All patients fulfilled the "UK Parkinson's Disease Society's Brain Bank Clinical Diagnostic Criteria" for idiopathic PD except that $28.2 \%$ reported one or more first, second, or third-degree relative diagnosed with PD [15]. Early onset (EO) was defined as being less than or equal to 50 years of age at diagnosis and late onset (LO) was defined as 51 years or more at diagnosis. As controls $(n=$ 509) we included neurologically healthy individuals visiting the Neurology Clinic, spouses of PD patients, anonymous blood donors, and individuals from the SNAC-K project (The Swedish National Study on Aging and Care in Kungsholmen, http://www.snac-k.se/), mean age was 71.8 years, and $57.3 \%$ were females (Table 1). Samples from patients and controls were collected after informed consent and approval of the local ethics committee Stockholm. DNA was obtained from blood samples using standard protocols. Blood from patients and control individuals randomly selected from the individuals recruited at the Neurology Clinic was used for EpsteinBarr virus (EBV) transfection of B-lymphocytes, EO patients $(n=10)$, LO patients $(n=10)$, and controls $(n=10)$. All investigations on human subjects were carried out following the rules of the Declaration of Helsinki of 1975, revised in 2008. A subset of the patients $(n=330)$ and controls $(n=317)$ was previously genotyped in a preliminary study published in a doctoral thesis [16].

2.2. Genotyping by Pyrosequencing and $q P C R$. We genotyped four SNPs in NRF2: rs35652124, rs6706649, rs6721961 in the promoter, and rs2001350. Genotyping of the three promoter SNPs was performed by pyrosequencing [17]. Rs2001350 was genotyped by TaqMan ${ }^{\circledR}$ quantitative real-time PCR (qPCR). For pyrosequencing, a PCR was run using Taq polymerase enzyme and two oligonucleotide primers in order to isolate 
a sequence of 209 base pairs containing the three promoter SNPs; forward primer, $5^{\prime}$-GAATGGAGACACGTGGGAGT$3^{\prime}$; and reverse primer, $5^{\prime}$-ACTTTACCGCCCGAGAATG- $3^{\prime}$ (Thermo Fisher Scientific, Hägersten, Sweden). The forward primer was biotinylated at the $5^{\prime}$ end. The PCR was programmed as follows: denaturation, at $95^{\circ} \mathrm{C}$ for 5 minutes, 45 cycles of amplification at $95^{\circ} \mathrm{C}$ for $20 \mathrm{sec}, 57^{\circ} \mathrm{C}$ for $20 \mathrm{sec}$, and $72^{\circ} \mathrm{C}$ for $30 \mathrm{sec}$, and terminal elongation at $72^{\circ} \mathrm{C}$ for 7 minutes. The forward strand of the PCR product was captured and isolated on streptavidin coated sepharose beads and then purified according to manufacturer's instructions using a filter-probe vacuum prep-tool. The single stranded DNA fragment was incubated with a sequencing primer and annealed for $2 \mathrm{~min}$ at $80^{\circ} \mathrm{C}$. SNPs rs35652124 and rs6706649 could be sequenced with the same sequence primer, $5^{\prime}$ TCACCCTGAACGC- ${ }^{\prime}$, and rs6721961 was sequenced with sequencing primer $5^{\prime}$-GGAGATGTGGACA- $3^{\prime}$. Sequences were analyzed using a pyrosequencing PSQ 96MA system (BIOTAGE AB, Uppsala, Sweden) using PyroMark Gold Q96 Reagents (QIAGEN Nordic, Sollentuna, Sweden). Rs2001350 was analyzed with TaqMan Fast 7500 qPCR. Rs2001350 was genotyped with a premade SNP genotyping assay, assay ID number C_11634985_10. We used a standard cycling program: pre-PCR reading at $60^{\circ} \mathrm{C}$ for one minute, polymerase activation at $95^{\circ} \mathrm{C}$ for 10 minutes, 55 cycles comprising denaturation at $92^{\circ} \mathrm{C}$ for 15 seconds and annealing/extension at $60^{\circ} \mathrm{C}$ for 1 minute, and last post-PCR reading at $60^{\circ} \mathrm{C}$ for 1 minute. TaqMan genotyping mix and assays were purchased from Applied Biosystems/Life Technologies (Life Technologies Europe BV, Stockholm, Sweden).

2.3. EBV Transfection of Lymphocytes. B-Lymphocytes were isolated from full blood using Ficoll-Paque according to manufacturer's instructions (GE Healthcare Bio-Sciences Corp., Piscataway, NJ, USA). Lymphocytes were cultivated in RPMI 1640 medium (SIGMA, St. Louis, MO, USA) with $20 \%$ fetal calf serum, L-glutamine $(200 \mathrm{mM})$, penicillin-streptomycin $(5000 \mu \mathrm{g} / \mathrm{mL}$ ) (Invitrogen, Carlsbad, CA, USA), and cyclosporine $(1 \mu \mathrm{L} / \mathrm{mL}$, Apoteket, Stockholm, Sweden). Transfection was achieved by adding and filtering supernatant of Epstein-Barr virus (EBV) infected B95-8 cells to the medium. When immortalized, cells were frozen and kept at $-140^{\circ} \mathrm{C}$ until use [18].

2.4. Reverse Transcription $q P C R$ ( $q R T-P C R)$. EBV transfected B-lymphocytes were thawed, reseeded, and harvested when cell count reached around 5 million cells and kept in $-130^{\circ} \mathrm{C}$. RNA was extracted from frozen cells using RNeasy Mini Kit according to manufacturer's instructions (QIAGEN Nordic, Sollentuna, Sweden). RNA concentration was measured and DNA contamination was eliminated. In order to avoid sample degradation, stable cDNA was synthesized from the RNA using a QuantiTect Reverse Transcription Kit (QIAGEN Nordic). The cDNA template was used for quantifying gene expression of NRF2 in PD patients and controls by qRT-PCR. Gene specific primers were designed for exon four of $N R F 2$, which is present in all mRNA transcripts: forward primer $5^{\prime}$-CTTTTGGCGCAGACATTCC$3^{\prime}$ and reverse primer $5^{\prime}$-AAGACTGGGCTCTCGATGTG$3^{\prime}$. GAPDH was used as housekeeping gene: forward primer $5^{\prime}$-AGCCACATCGCTCAGACA- $3^{\prime}$ and reverse primer $5^{\prime}$ GCCCAATACGACCAAATCC-3' (Thermo Fisher Scientific). NRF2 primer pair gave an $R^{2}$ value of 0.9507 and GAPDH primes gave an $R^{2}$ value of 0.9809 . The qRT-PCR reaction was performed according to standard protocols, using triplicates of each sample and SYBR green qRT-PCR mastermix from Applied Biosystems (Applied Biosystems, Life Technologies Europe BV, Stockholm, Sweden). The cycler was programmed as follows: holding stage for pre-PCR read and enzyme activation: $50^{\circ} \mathrm{C}$ for $2 \mathrm{~min}$ and $95^{\circ} \mathrm{C}$ for $10 \mathrm{~min}$, amplification for 45 cycles: $95^{\circ} \mathrm{C}$ for $15 \mathrm{sec}, 60^{\circ} \mathrm{C}$ for $1 \mathrm{~min}$, and last melting curve and post-PCR read: $95^{\circ} \mathrm{C}$ for $15 \mathrm{sec}, 60^{\circ} \mathrm{C}$ for $1 \mathrm{~min}, 95^{\circ} \mathrm{C}$ for $30 \mathrm{sec}$, and $60^{\circ} \mathrm{C}$ for $15 \mathrm{sec}$.

2.5. Statistical Analysis and Image Analysis. Genotype and allele frequencies were compared between PD patients and controls. Association for genotypes was evaluated with Chi square $\left(\mathrm{X}^{2}\right)$ test and allele association was analyzed using Fisher's exact test. We used GraphPad Prism 5.03 (GraphPad Software Inc., La Jolla, CA, USA) for the analysis, significance level of $5 \%$ and two-sided $p$ values. Bonferroni correction was used to correct for multiple testing. To control for the skewed gender distribution in the patient group (males $=63.3 \%$ ), genotype analysis was verified using a logistic regression with gender as cofactor; analysis was run in PLINK v1.07 [19]. Hardy-Weinberg equilibrium was evaluated using a free online software through a $\mathrm{X}^{2}$ based test [20]. Haplotype analysis was run using the software Haploview4.2 [21]. qRTPCR data was analyzed in qBase 1.3.3, a software used for automated analysis of real-time quantitative PCR data [22], and evaluated with a student's $t$-test or a one-way ANOVA using GraphPad Prism 5.03.

\section{Results}

3.1. Genotyping by Pyrosequencing and $q P C R$. We have genotyped four common SNPs in the NRF2 gene in 504 PD patients from the Stockholm area of Sweden and 509 geographically matched control individuals. One genetic variation, rs2001350, lies in the large second intron of the gene; the three other SNPs, rs35652124, rs6706649, and rs6721961, are promoter SNPs. Results for the genotype and allele analysis can be found in Table 2. Genotype and allele association analysis revealed no significant associations with $\mathrm{PD}$. All four SNPs were in Hardy-Weinberg equilibrium (data not shown). The logistic regression, which included gender as a covariate, was run under a genotypic model with 2 degrees of freedom confirming the results from the genotype association analysis (supplementary table 1 in Supplementary Material available online at https://doi.org/10.1155/2017/4020198), verifying that there was no bias introduced by the skewed gender distributions in the patient and control group. In order to analyze the combined effect of several SNPs in NRF2, we further ran a haplotype analysis, which did not reveal any association between these four SNPs and PD (supplementary table 2). 
TABLE 2: Results from genotype and allele analysis.

\begin{tabular}{|c|c|c|c|c|c|}
\hline & Controls\% (n) & $\mathrm{PD} \%(n)$ & $\mathrm{X}^{2}(\mathrm{DF})$ & OR (95\% CI) & $p$ value \\
\hline \multicolumn{6}{|c|}{$r s 35652124$} \\
\hline $\mathrm{AA}$ & $47.7(230)$ & $45.0(215)$ & $0.79(2)$ & & 0.68 \\
\hline AG & $43.6(210)$ & $45.4(217)$ & & & \\
\hline GG & $8.7(42)$ & $9.6(46)$ & & & \\
\hline A & $69.5(670)$ & $67.7(647)$ & & $1.09(0.90-1.32)$ & 0.40 \\
\hline G & $30.5(294)$ & $32.3(309)$ & & & \\
\hline \multicolumn{6}{|c|}{$r s 6706649$} \\
\hline GG & $80.0(387)$ & $80.2(385)$ & $0.011(2)$ & & 0.99 \\
\hline GA & $18.6(90)$ & $18.3(88)$ & & & \\
\hline $\mathrm{AA}$ & $1.4(7)$ & $1.5(7)$ & & & \\
\hline G & $89.3(864)$ & $89.4(858)$ & & $0.99(0.74-1.32)$ & 0.94 \\
\hline $\mathrm{A}$ & $10.7(104)$ & $10.6(102)$ & & & \\
\hline \multicolumn{6}{|c|}{ rs6721961 } \\
\hline CC & $74.8(365)$ & $77.4(370)$ & $2.39(2)$ & & 0.30 \\
\hline CA & $24.6(120)$ & $21.3(102)$ & & & \\
\hline $\mathrm{AA}$ & $0.6(3)$ & $1.3(6)$ & & & \\
\hline $\mathrm{C}$ & $87.1(850)$ & $88.1(842)$ & & $0.91(0.70-1.20)$ & 0.54 \\
\hline $\mathrm{A}$ & $12.9(126)$ & $11.9(114)$ & & & \\
\hline \multicolumn{6}{|c|}{ rs2001350 } \\
\hline AA & $80.5(388)$ & $79.5(387)$ & $2.66(2)$ & & 0.26 \\
\hline AG & $19.3(93)$ & $19.5(95)$ & & & \\
\hline GG & $0.2(1)$ & $1.0(5)$ & & & \\
\hline A & $90.1(869)$ & $89.2(869)$ & & $1.12(0.82-1.48)$ & 0.55 \\
\hline G & $9.9(95)$ & $10.8(105)$ & & & \\
\hline
\end{tabular}

$n$ : number of individuals; PD: Parkinson's disease; $\mathrm{X}^{2}$ : chi square; DF: degrees of freedom; OR: odds ratio; 95\% CI: 95\% confidence interval.

TABLE 3: Results from age stratified genotype and allele analysis for rs35652124.

\begin{tabular}{|c|c|c|c|c|c|}
\hline rs35652124 & Controls\% (n) & LO PD\% $(n)$ & $\mathrm{EO}$ PD\% $(n)$ & $\mathrm{X}^{2}(\mathrm{DF})$ & $p$ value \\
\hline $\mathrm{AA}$ & $47.7(230)$ & $41.9(163)$ & $58.4(52)$ & $14.19(4)$ & 0.0067 \\
\hline AG & $43.6(210)$ & 49.4 (192) & $28.1(25)$ & & \\
\hline GG & $8.7(42)$ & $8.7(34)$ & $13.5(12)$ & & \\
\hline A & $69.5(670)$ & $66.6(518)$ & 72.5 (129) & $3.82(2)$ & 0.15 \\
\hline G & $30.5(294)$ & $33.4(260)$ & $27.5(49)$ & & \\
\hline
\end{tabular}

$n$ : number of individuals; PD: Parkinson's disease; LO: late onset; EO: early onset; $\mathrm{X}^{2}$ : chi square; DF: degrees of freedom.

When patients were stratified in groups of $\mathrm{EO}$ or LO, genotype association analysis showed that one of the promoter SNPs, rs35652124, was significantly associated with EO $\mathrm{PD}$ when compared to controls $\left(\mathrm{X}^{2}=14.19 p=0.0067\right)$, (Table 3); both the WT genotype AA and the mutated genotype GG of rs35652124 were observed more frequently in PD patients with EO than in individuals in the two other groups, while EO heterozygous carriers were significantly fewer. The significance remained after correction for multiple testing, $p=0.027$. The association was dissected further, which showed that the significance was dependent on the group of patients with early onset. The significance remained when comparing controls to EO patients only $(p=0.019)$ and when comparing LO patients to EO patients ( $p=0.0013)$, but not when comparing controls to LO patients $(p=0.20)$. An additional analysis comparing patients with EO PD against
LO PD and controls under a recessive model (AA + GA versus $G G$ ) and a dominant model (AA versus $G A+G G$ ), respectively, confirmed the association under a dominant model $\left(\mathrm{X}^{2}=6.68, p=0.013\right)$ and enabled us to establish the causality between the absence of G alleles and EO PD. Allele frequencies for rs35652124 were not associated with age at diagnosis $(p=0.15)$, nor did genotype or allele for rs6706649, rs6721961, and rs200350 (supplementary table 3).

3.2. mRNA Expression Analysis. Since promoter SNPs in NRF2 have been suggested to influence gene expression in vitro, we further wanted to investigate whether gene expression correlated with the rs35652124 genotype [23]. We performed qRT-PCR on a subset of the patients included in our genetic study; mRNA levels were normalized to GAPDH expression and to a reference sample consisting of mixed 


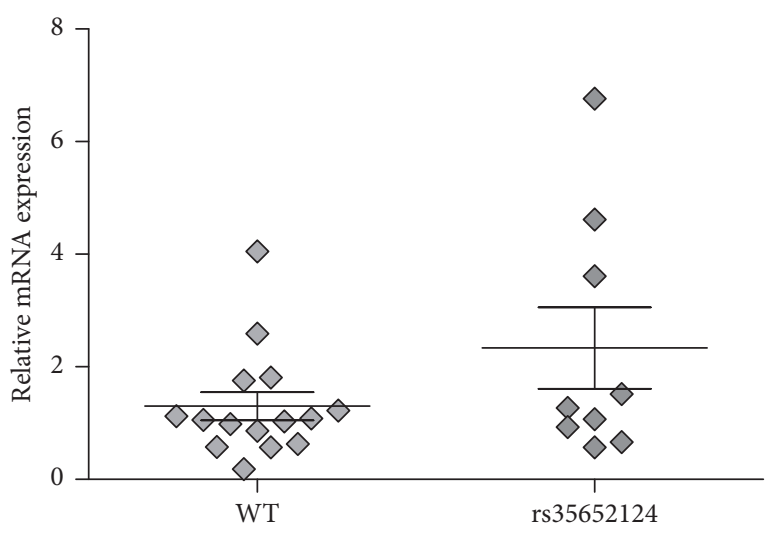

(a)

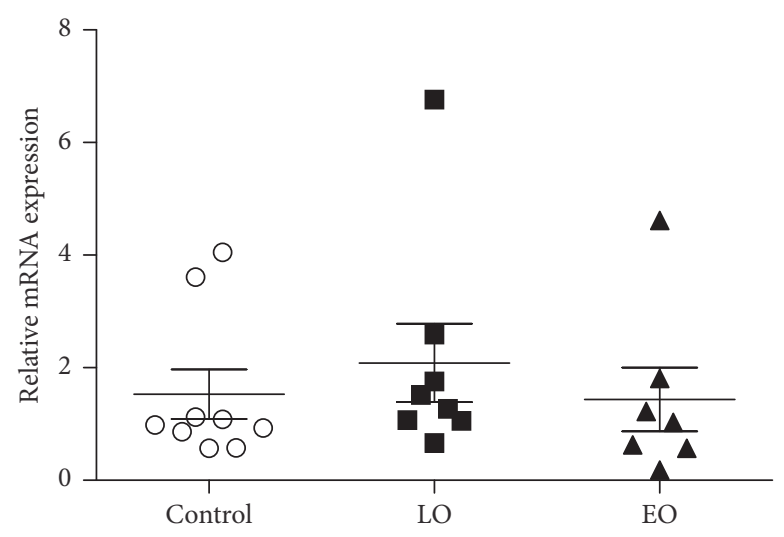

(b)

FIGURE 1: rs35652124 genotype and age of onset do not correlate with NRF2 mRNA expression. NRF2 mRNA expression in EBV transfected human lymphocytes. Values were normalized to GAPDH mRNA and to a reference sample consisting of cDNA from all control individuals; groups were compared using a $t$-test (a) or one-way ANOVA (b) analysis. Error bars: standard error of mean. In (a) mRNA expression of all subjects was divided into two groups depending on their genotype for the promoter SNPs rs35652124. WT: subjects wild type for rs35652124 and rs35652124; subjects heterozygous $(n=8)$ and homozygous $(n=1)$ for rs35652124, $p=0.121$. In (b) mRNA levels are analyzed according to disease status; LO: late onset PD; EO: early onset PD; $p=0.695$.

cDNA from all control individuals. mRNA expression in wild-type (WT) carriers of the promoter SNP rs35652124 $(n=$ $15)$ was compared to individuals carrying one $(n=8)$ or two $(n=1)$ mutated alleles at this position. We found no significant difference between the carriers and noncarriers, (Student's $t$-test, $p=0.121$ ) (Figure $1(\mathrm{a})$ ). It is noteworthy that the individual with the highest relative NRF2 expression (6.76) was homozygous GG for rs35652124 but carried the WT allele of the three other SNPs; exact mRNA levels and genotypes are found in supplementary table 4 . We additionally compared NRF2 mRNA levels in healthy controls $(n=9)$ and PD patients $(n=15)$ and found no significant difference $(p=0.712$, supplementary table 4$)$. Also when stratifying the material into two patient groups with LO PD $(n=8)$ or EO PD $(n=7)$ expression levels were similar in all three groups $(p=0.695)$ (Figure 1(b), supplementary table 4$)$. NRF2 mRNA levels in these individuals were quite variable, displaying a few higher expressing individuals in all groups. Last we analyzed the expression data with respect to age using linear regression (Figure 2, supplementary table 4). We found no correlation between expression levels of NRF2 and age either when analyzing the entire sample regardless of disease status ( $p=0.799)$ or in controls alone $(p=0.429)$.

\section{Discussion}

We have found a genotype association of rs35652124 with EO PD. Genotype frequencies for this SNP were difficult to interpret, as the EO patients were more often homozygous for both the wild-type and mutant allele. This could possibly mean that the mode of penetrance of this genetic variation is unlikely to be additive or multiplicative. When combining the genotypes, we found that both genotypes comprising the mutated allele, heterozygous GA or homozygous GG, were more common in the control group and PD patients with late onset, which is consistent with the minor allele $\mathrm{G}$ being

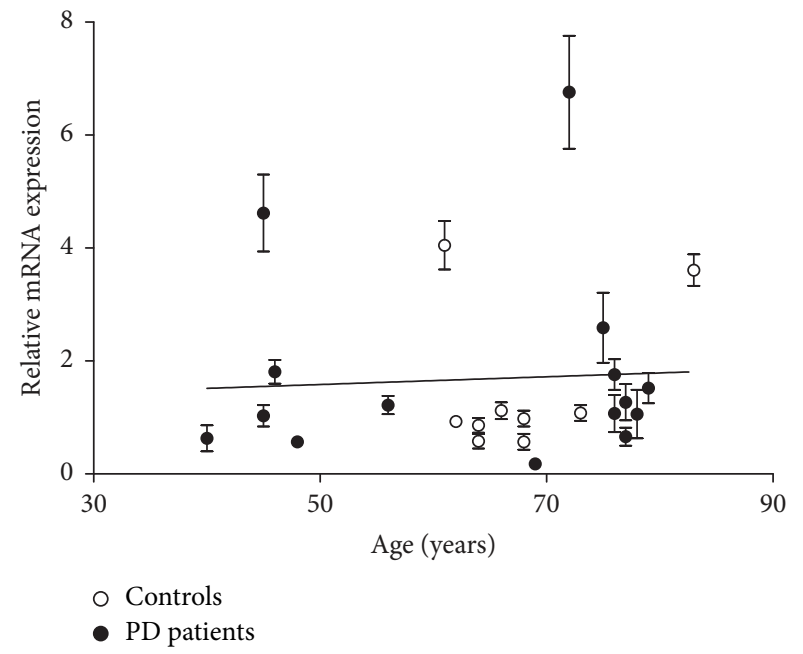

FIGURE 2: mRNA levels do not correlate with age. NRF2 mRNA expression in EBV transfected lymphocytes from individuals of different age spanning from 40 years to 80 ; PD patients are represented in black and controls in white. Each data point corresponds to the mean value of triplicates normalized to GAPDH and to a reference sample consisting of cDNA from all control individuals; error bars: standard error of mean. Correlation between age and expression of NRF2 was evaluated using linear regression and was found nonsignificant ( lope $=0.0068, p=0.799$ ) when the entire material was analyzed regardless of disease and when controls were analyzed separately (regression line not shown, slope $=0.060, p=0.429$ ).

rarer in the early onset population. Analyzing the material under a dominant model confirmed the association between the absence of $G$ alleles and earlier disease onset. We therefore stipulate that the $\mathrm{G}$ allele confers some protection against $\mathrm{PD}$ by delaying the onset of symptoms. von Otter et al. previously reported a promoter haplotype consisting of the wild-type 
alleles of rs35652124, rs6706649, and rs6721961 associated with later age of onset and less risk of developing disease [11]. Together, these datasets point to a disease modifying role for promoter SNPs, in particular rs35652124.

In vitro data indicate that the promoter SNPs analyzed by us could influence the transcriptional activity of NRF2 $[23,24]$. The mutated allele of rs6706649 and rs6721961 has been reported to cause a drastic decrease of NRF2 gene transcription in transfected cells, while decrease is less pronounced when the mutated allele of rs35652124 is combined with the mutated allele of rs6706649. This information, together with the results from our genetic analysis, prompted us to investigate the functional implication of the rs35652421 SNP in human tissue [23, 24]. NRF2 mRNA expression was quantified using qRT-PCR in patient specific cell-lines. Although qRT-PCR analysis showed no significant differences in mRNA levels when subjects were stratified according to genotype, quantification also revealed that NRF2 expression levels were quite variable in both groups, and the standard error of mean in these groups was consequently large. Further analysis of gene expression data did not reveal any difference between control individuals and PD patients or between groups of patients receiving a diagnosis before and after the age of 50 years. There was still great variability between individuals within all groups. Furthermore, the variable expression levels did not correlate with the age of the study participants (spanning from 40 to 83 years), which is surprising considering previous reports of Nrf2 decreasing with increasing age $[4,6]$. The marked divergence in expression levels within the different groups in our study indicates that there is a regulatory parameter not included in our analysis. We might therefore hypothesize that there are more regulatory NRF2 SNPs which could influence the gene expression of NRF2, independently or in concert with rs35652421.

\section{Conclusions}

We have found a genotype association of rs35652124 with EO $\mathrm{PD}$, a finding which supports the involvement of NRF2 in PD. Our results indicate that NRF2 has a disease modifying effect rather than affecting disease risk, but more detailed studies of the NRF2 gene in larger populations are warranted. Quantification of mRNA in human blood cells showed that NRF2 expression in control subjects and that of PD patients were similar and that the promoter SNP rs35652124 does not have a consistent effect on mRNA levels. Thus far, there is sparse functional evidence available to illuminate the role of genetic variants in NRF2 in PD. Therefore it is also important to expand the knowledge on the function of NRF2 in pathophysiology in order to conclude on the importance of this gene in PD.

\section{Conflicts of Interest}

The authors declare that there are no conflicts of interest regarding the publication of this paper.

\section{Acknowledgments}

The authors would like to thank Laura Fratiglioni for providing them with control samples from the SNAC-K project. Postmortem human tissue was provided by the Netherlands Brain Bank (Amsterdam, Netherlands). This study was supported by the Swedish Brain Power, the Swedish Research Council, the Swedish Parkinson Foundation, the Swedish Brain Foundation, Åke Wibergs Stiftelse, and Karolinska Institutet Funds.

\section{References}

[1] C. Ran and A. C. Belin, “The genetics of Parkinson's disease: review of current and emerging candidates," Journal of Parkinsonism and Restless Legs Syndrome, vol. 2014, no. 4, pp. 63-75, 2014.

[2] P. Moi, K. Chan, I. Asunis, A. Cao, and Y. W. Kan, "Isolation of NF-E2-related factor 2 (Nrf2), a NF-E2-like basic leucine zipper transcriptional activator that binds to the tandem NF-E2/AP1 repeat of the $\beta$-globin locus control region," Proceedings of the National Academy of Sciences of the United States of America, vol. 91, no. 21, pp. 9926-9930, 1994.

[3] T. W. Kensler, N. Wakabayashi, and S. Biswal, "Cell survival responses to environmental stresses via the Keap1-Nrf2-ARE pathway," Annual Review of Pharmacology and Toxicology, vol. 47, pp. 89-116, 2007.

[4] P.-H. Shih and G.-C. Yen, "Differential expressions of antioxidant status in aging rats: the role of transcriptional factor Nrf2 and MAPK signaling pathway," Biogerontology, vol. 8, no. 2, pp. 71-80, 2007.

[5] K. Itoh, T. Chiba, S. Takahashi et al., "An Nrf2/small Maf heterodimer mediates the induction of phase II detoxifying enzyme genes through antioxidant response elements," Biochemical and Biophysical Research Communications, vol. 236, no. 2, pp. 313-322, 1997.

[6] J. H. Suh, S. V. Shenvi, B. M. Dixon et al., "Decline in transcriptional activity of Nrf2 causes age-related loss of glutathione synthesis, which is reversible with lipoic acid," Proceedings of the National Academy of Sciences of the United States of America, vol. 101, no. 10, pp. 3381-3386, 2004.

[7] C. P. Ramsey, C. A. Glass, M. B. Montgomery et al., "Expression of Nrf2 in neurodegenerative diseases," Journal of Neuropathology and Experimental Neurology, vol. 66, no. 1, pp. 75-85, 2007.

[8] Y.-C. Chen, Y.-R. Wu, Y.-C. Wu, G.-J. Lee-Chen, and C.M. Chen, "Genetic analysis of NFE2L2 promoter variation in Taiwanese Parkinson's disease," Parkinsonism and Related Disorders, vol. 19, no. 2, pp. 247-250, 2013.

[9] Y. Gui, L. Zhang, W. Lv, W. Zhang, J. Zhao, and X. Hu, "NFE2L2 variations reduce antioxidant response in patients with Parkinson disease," Oncotarget, vol. 7, no. 10, pp. 1075610764, 2016.

[10] M. Todorovic, J. R. B. Newman, J. Shan et al., "Comprehensive assessment of genetic sequence variants in the antioxidant 'master regulator' Nrf2 in idiopathic parkinson's disease," PLoS ONE, vol. 10, no. 5, Article ID e0128030, 2015.

[11] M. von Otter, S. Landgren, S. Nilsson et al., "Association of Nrf2encoding NFE2L2 haplotypes with Parkinson's disease," BMC Medical Genetics, vol. 11, no. 1, article 36, 2010.

[12] M. von Otter, P. Bergström, A. Quattrone et al., "Genetic associations of Nrf2-encoding variants with Parkinson's disease - a multicenter study," BMC Medical Genetics, vol. 15, no. 1, article 131, 2014. 
[13] M. von Otter, S. Landgren, S. Nilsson et al., "Nrf2-encoding NFE2L2 haplotypes influence disease progression but not risk in Alzheimer's disease and age-related cataract," Mechanisms of Ageing and Development, vol. 131, no. 2, pp. 105-110, 2010.

[14] P. Bergström, M. Von Otter, S. Nilsson et al., "Association of NFE2L2 and KEAP1 haplotypes with amyotrophic lateral sclerosis," Amyotrophic Lateral Sclerosis and Frontotemporal Degeneration, vol. 15, no. 1-2, pp. 130-137, 2014.

[15] S. E. Daniel and A. J. Lees, "Parkinson's Disease Society Brain Bank, London: overview and research," Journal of Neural Transmission. Supplementa, vol. 39, pp. 165-172, 1993.

[16] C. Ran, On Genes Involved in Common Neurological Disorders: Focus on Parkinson's Disease. Karolinska Institutet, Sweden, Stockholm, 2014.

[17] M. Ronaghi, M. Uhlén, and P. Nyrén, "A sequencing method based on real-time pyrophosphate," Science, vol. 281, no. 5375, pp. 363-365, 1998.

[18] E. V. Walls, M. G. Doyle, K. K. Patel, M. J. Allday, D. Catovsky, and D. H. Crawford, "Activation and immortalization of leukaemic B cells by Epstein-Barr virus," International Journal of Cancer, vol. 44, no. 5, pp. 846-853, 1989.

[19] S. Purcell, B. Neale, K. Todd-Brown et al., "PLINK: a tool set for whole-genome association and population-based linkage analyses," American Journal of Human Genetics, vol. 81, no. 3, pp. 559-575, 2007.

[20] S. Rodriguez, T. R. Gaunt, and I. N. M. Day, "Hardy-Weinberg equilibrium testing of biological ascertainment for Mendelian randomization studies," American Journal of Epidemiology, vol. 169, no. 4, pp. 505-514, 2009.

[21] J. C. Barrett, B. Fry, J. Maller, and M. J. Daly, "Haploview: analysis and visualization of LD and haplotype maps," Bioinformatics, vol. 21, no. 2, pp. 263-265, 2005.

[22] J. Hellemans, G. Mortier, A. de Paepe, F. Speleman, and J. Vandesompele, "qBase relative quantification framework and software for management and automated analysis of real-time quantitative PCR data," Genome Biology, vol. 8, no. 2, p. R19, 2007.

[23] J. M. Marzec, J. D. Christie, S. P. Reddy et al., "Functional polymorphisms in the transcription factor NRF2 in humans increase the risk of acute lung injury," FASEB Journal, vol. 21, no. 9, pp. 2237-2246, 2007.

[24] C.-C. Hua, L.-C. Chang, J.-C. Tseng, C.-M. Chu, Y.-C. Liu, and W.-B. Shieh, "Functional haplotypes in the promoter region of transcription factor Nrf2 in chronic obstructive pulmonary disease," Disease Markers, vol. 28, no. 3, pp. 185-193, 2010. 


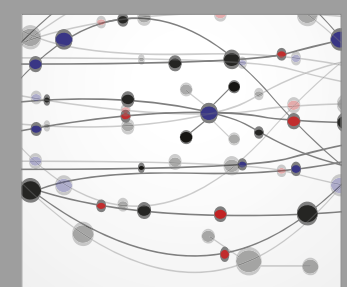

The Scientific World Journal
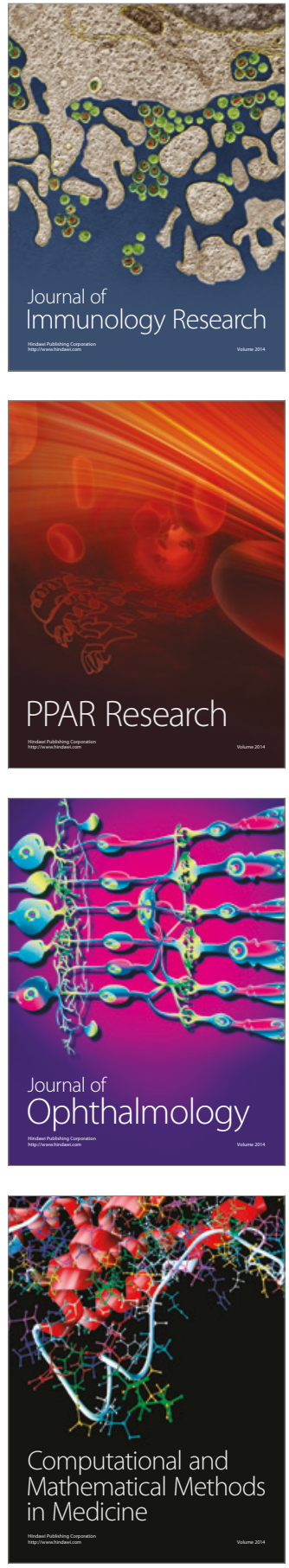

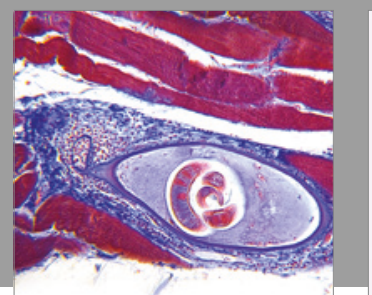

Gastroenterology Research and Practice
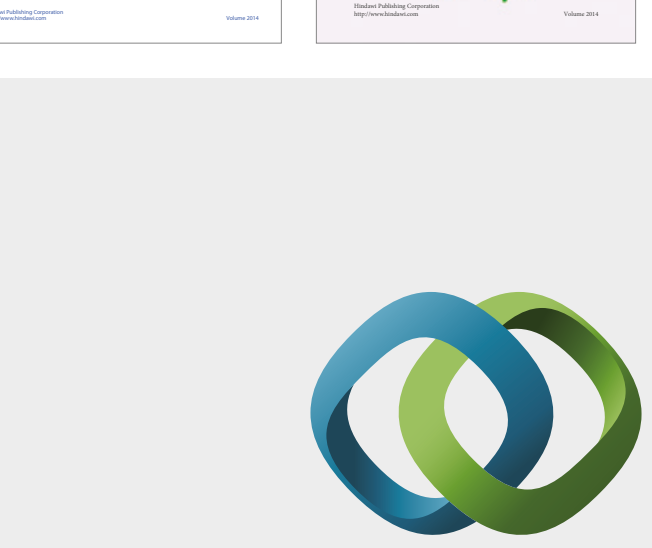

\section{Hindawi}

Submit your manuscripts at

https://www.hindawi.com
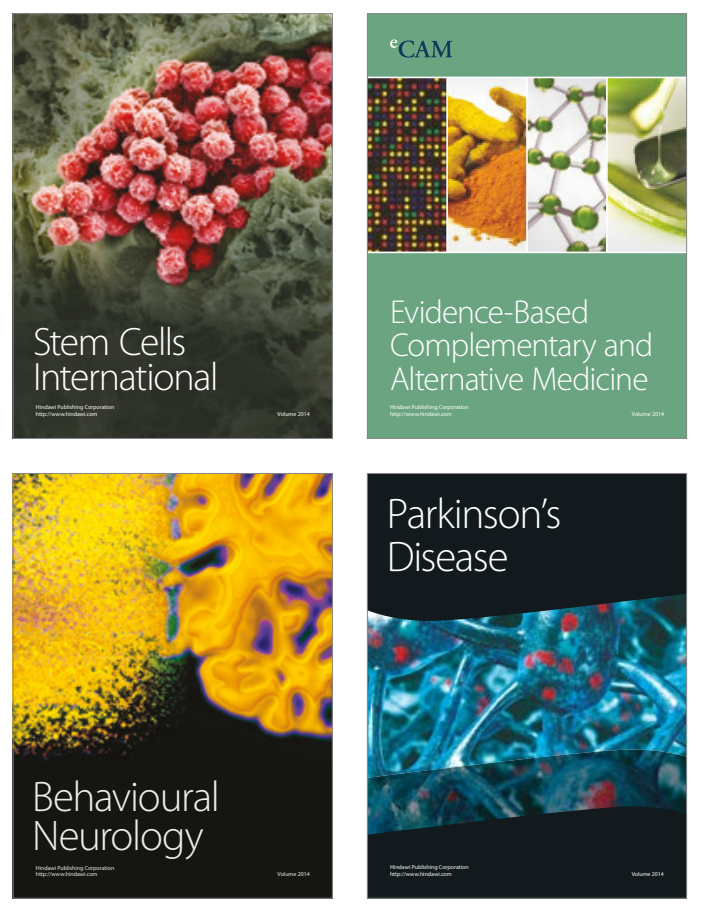
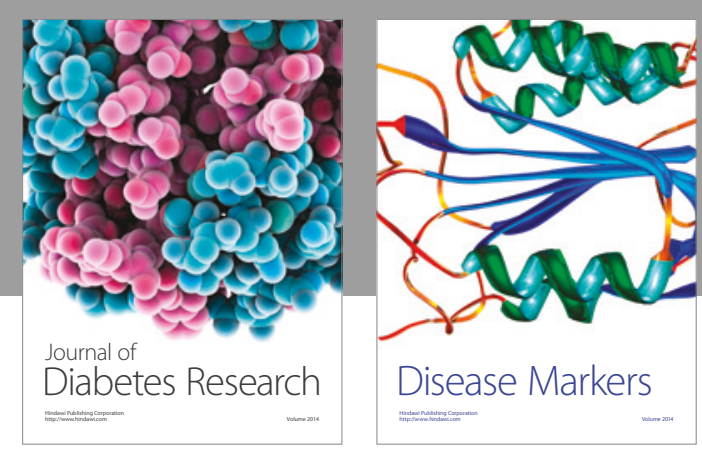

Disease Markers
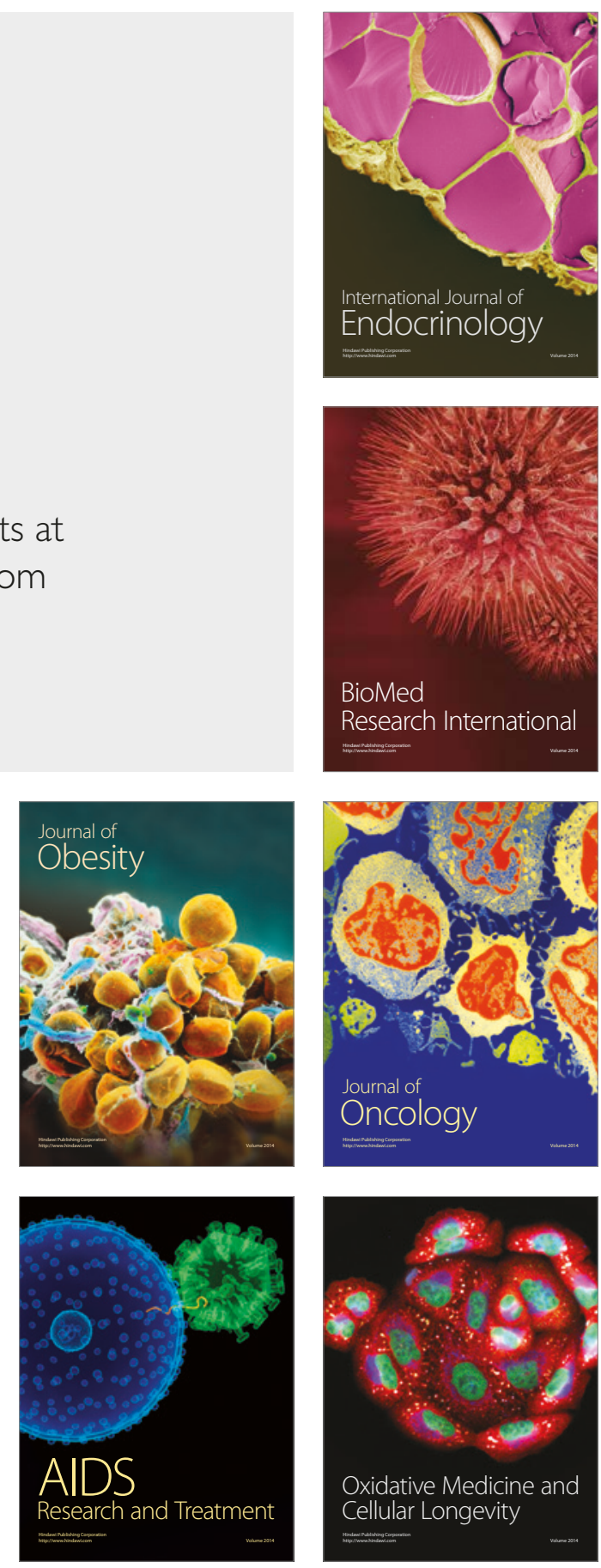